\title{
Research and Implementation of WebGIS Based on Silverlight
}

\author{
Jie Zhou ${ }^{1, a}$, Xiaoyan Sun ${ }^{1, b}$, Jinglin Du ${ }^{1, c}$ \\ ${ }^{1}$ College Of Electronics and Information Engineering,Nanjing University of Information Science and \\ Technology,Nanjing 210044, China \\ ajiezhou45@hotmail.com, bsun.xiaoyan.1989@163.com, cjldu@nuist.edu.cn
}

Keywords: Silverlight;Bing Maps;Web Service;GIS

\begin{abstract}
As the impact of meteorological information becomes more extensive, the demand of user application interface and interaction effect is increasingly high, the necessity of the application code portability gets more evident, we selected the cross-platform, cross-browser Web front-end technology-Silverlight, combined with WebService interface provided by Bing Maps, designed a Silverlight-based meteorological service system to make any user at any time, any place connected to the Internet, automatically acquire the real-time severe weather warning signals and other conventional weather forecast released by meteorological department. The system provides a WebService-based GIS function. Studies show that the combination of rich client and network services is feasible in the Web Map Service.
\end{abstract}

\section{Introduction}

Natural disasters, such as typhoon, could have an enormous impact on society. The level of the public's preparedness could make a significant difference in the severity of casualty and damage inflicted by such storms. It is affirmed that there is a critical need for the development of weather warning and forecast system products that give attention to storm-scale weather [1]. The recent development of GIS (Geographical Information Science) has facilitated its application on meteorological information services at high frequency. Many government and private agencies are working on its behavior but still it is challenging and incomplete.

The development of geospatial data and services blossoms in recent years. There have been potential solutions to integrate and interoperate these heterogeneous and widely-distributed geospatial resources. Due to the increasingly high demand for the user application interface and mutual effects [2], as well as the portability of application code has become more evident, to design a complete user interaction, conveniently portable meteorological early warning system is particularly important.

\section{System Design and Implementation}

System Development Environment. The platform is a combination of rich client technologySliverlight and back-end technology - WebService applied in GIS [3]. We use C \# and XAML to develop the Silverlight-based meteorological service system. The system uses Visual Studio 2008 as a background development tools, uses Bing Maps as a development platform, loads the meteorological data from oracle $10 \mathrm{~g}$ database.

Silverlight is a kind of cross-platform and cross-browser RIA (Rich Internet Application) technologies provided by Microsoft [4], which supports dot net language, and implement the integrated development mode with Visual Studio. As a new Web content carrier, when the client browser side runs, it not only can interact with a variety of server side applications neatly, but also has the desktop experience, rich graphics rendering and user interaction control capabilities. With the continuous development of Silverlight technology in Internet field, more and more developers, designers began to use this technology to achieve different kinds of Web applications. Here there is nolack of domestic well-known sites such as Baidu, Tencent and Taobao. 
Bing Maps was developed by Microsoft against Google Earth. It releases an online map service, supports users to develop and load to deploy their own map services, opens up a Silverlight and Ajax-based client development interface. Bing Maps provides free remote sensing images, which are basically taken after 2002, can cover most parts of China, and many major cities have been updated to 2008, whose resolution in some areas can reach 0.6m [5]. Bing Maps signed a data-sharing agreement with ESRI, and updates parts of the data every month, maintaining a good timeliness of map data. Bing Maps provides many rich and free data resources, how to use these resources is of great concern. In addition, it provides APIs that can be developed, user controls can freely customize the browser-based application system, you can use data services from service provider on the web.

System Architecture Design. The main purpose of this system is to present and process meteorological data, especially the historical typhoon data, so as to proffer meteorological disaster warning. It can dynamically display typhoon path on web pages. The system architecture is shown in Figure 1. The system timing diagram is shown in Figure 2.

This system adopts a Browser-Server architecture developed with C\# and XAML. The architecture makes the server side to do most data processing and computing and the client side to provide functions for interactions between users and the whole system. Interactive functions include data and service search, access, quality evaluation, visualization, and map controls. The designed principle is based on the three-tier application, which is perceived as the backbone of this architecture. User Interface Layer is designed on the client side, besides Data Access Layer is designed on the server side. But not all of the business logic is designed on the server side. Part of the business logic, mainly about GIS, is kept on the server side, in the meantime, rest of the business logic which we call as common business logic is offloaded onto the client side.

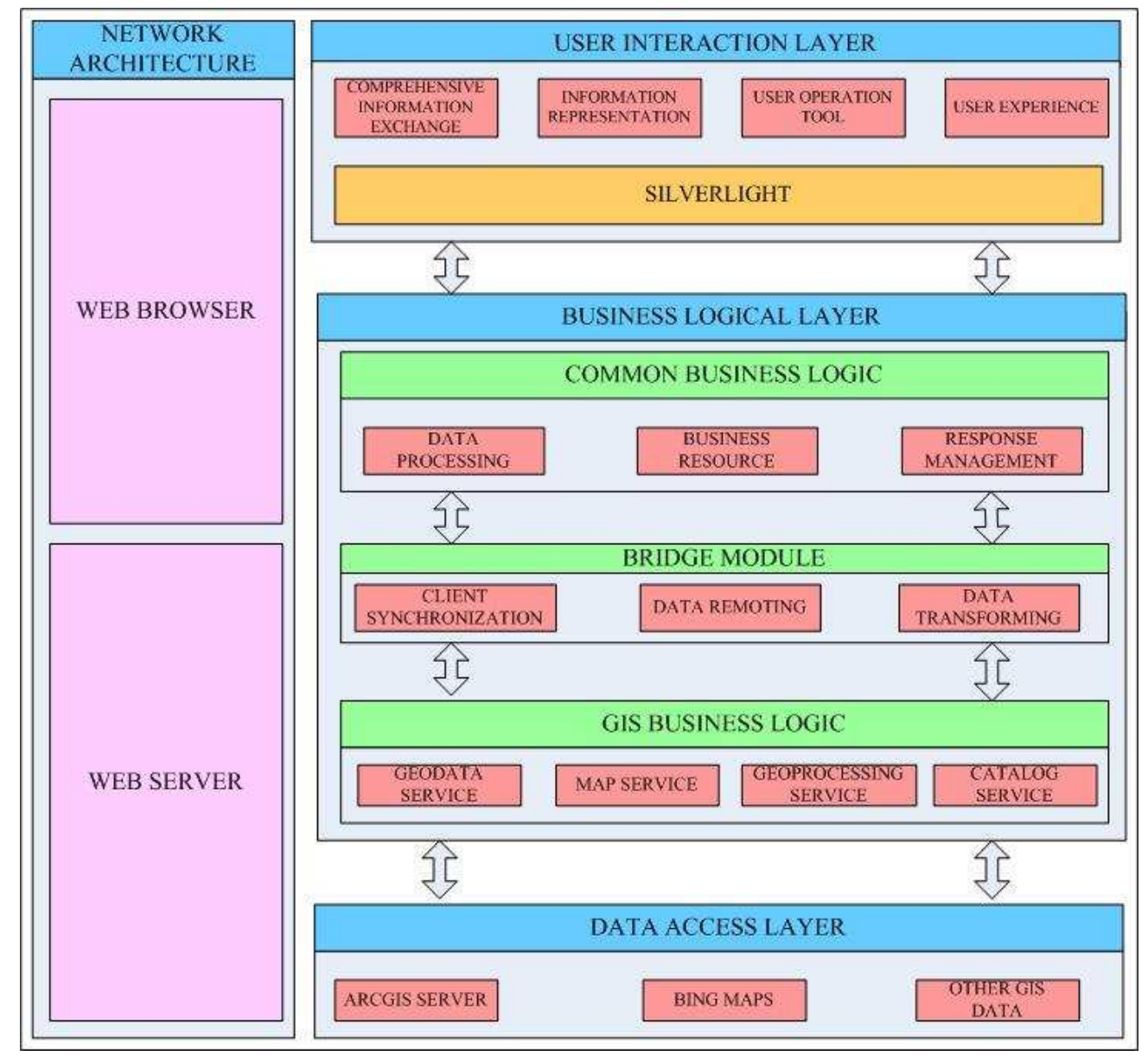

Fig. 1 System Architecture 


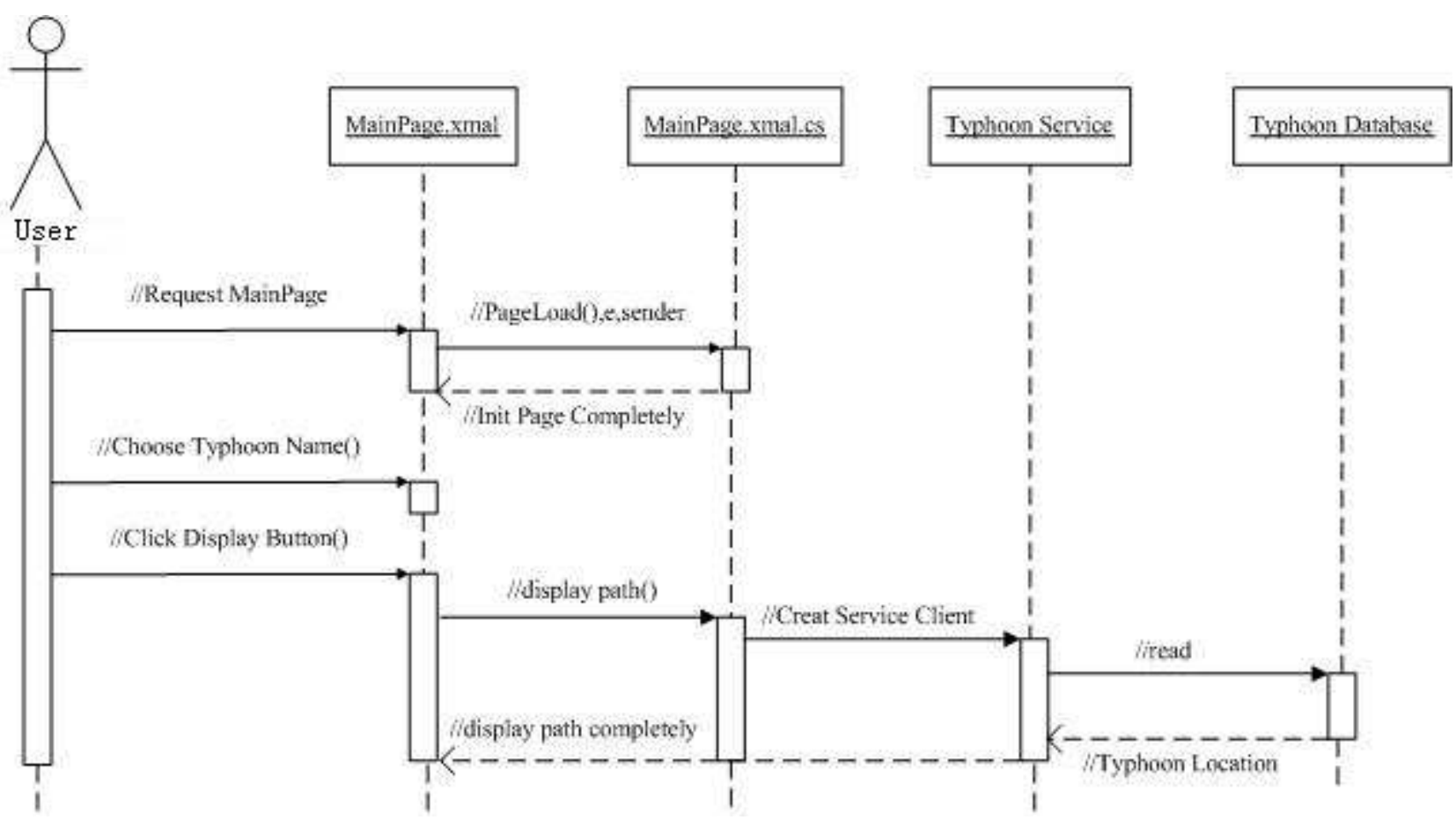

Fig. 2 System Timing Diagram

Database Analysis. All the meteorological data given are stored in oracle $10 \mathrm{~g}$ database. Take the table of typhoon as an example, it's shown in Figure 3. This table stores the information of typhoon center latitude and longitude position, central pressure, maximum wind speed in the past 12 hours and coming 96 hours at intervals of 6 hours. In order to display typhoon path on Bing Maps, read the latitude and longitude of the typhoon center from data table.

\begin{tabular}{|l|l|l|l|}
\hline \multicolumn{1}{|c|}{ Name } & \multicolumn{1}{|c|}{ Type } & Nullable & \multicolumn{1}{c|}{ Comments } \\
\hline YYGGGG & NUMBER (12) & & Published Time \\
\hline TT & VARCHAR2 (10) & $\sqrt{ }$ & Cyclone Level (TY STS、 TS) \\
\hline NAME & VARCHAR2 (10) & $\sqrt{ }$ & Cyclone Name \\
\hline NNMN & VARCHAR2 (10) & $\sqrt{ }$ & National Cyclone Number \\
\hline N1N1N1N1 & VARCHAR2 (10) & $\sqrt{ }$ & International Cyclone Number \\
\hline Y1Y1G1G1G1G1 & NUMBER $(6)$ & $\sqrt{ }$ & Real Time of Cyclone \\
\hline LALA00 & NUMBER $(6,2)$ & $\sqrt{ }$ & Latitude of Cyclone \\
\hline LOL000 & NUMBER $(6,2)$ & $\sqrt{ }$ & Longitude of Cyclone \\
\hline PPP00 & NUMBER $(6,2)$ & $\sqrt{ }$ & Central Air Pressure of Cyclone \\
\hline FF00 & NUMBER $(6,2)$ & $\sqrt{ }$ & Maximum Wind Speed \\
\hline RR30 & NUMBER $(6,2)$ & $\sqrt{ }$ & Wind Radii 30 \\
\hline RR50 & NUMBER $(6,2)$ & $\sqrt{ }$ & Wind Radii 50 \\
\hline DDD12 & VARCHAR2 $(6)$ & $\sqrt{ }$ & Direction of Movement \\
\hline
\end{tabular}

Fig.3 Part of Typhoon Table

Application of WebService. Bing Maps supplies a set of WCF (Windows Communication Foundation) map services to users. Base on these map services, we achieve the completion of corresponding functions [6]. When using the service, create a service request first, set request conditions, the request is conveyed to Bing Maps service center. The Web server responses the request, allows the asynchronous call to the relevant Web service and requests the execution results back to the client. 
The Silverlight-based meteorological service system we designed uses GeocodeService [7] to achieve the location search function and anti-search function. When users input address, it returns the corresponding coordinates; otherwise, when users input coordinates, it shows the output address through adding pushpin.

After obtaining the typhoon path point coordinates (longitude, latitude) by calling the WebService, the path circuit diagram is drawn on Bing Maps and circular mark is put on the path points. Pop-up message box displays when the mouse is moved into the round tag. The typhoon's central pressure, maximum wind speed and other information will be shown; the message box is hidden when the mouse is removed. Typhoon path is displayed as shown in Figure 4. The display helps the user to observe at a glance to the typhoon movements, not only can see the historical typhoon path, but also can query typhoon center in the future period of time. It does some warnings to people's daily live.

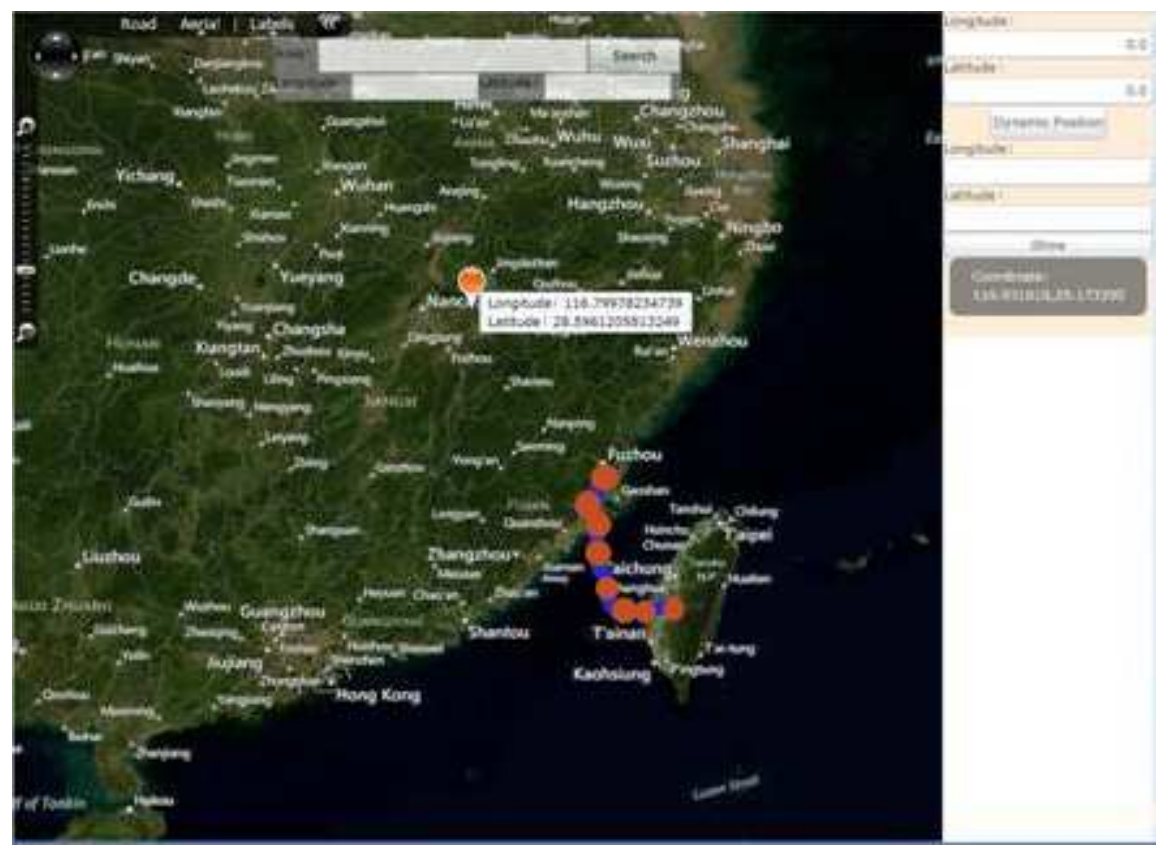

Fig.4 Typhoon Path Display

\section{Conclusion}

The paper founded on Silverlight and WebService applied research in GIS, explored GIS rich media network applications which combines the Web front-end technology-Silverlight with back-end technology-WebService, tried to complete not only the artistic interface design, rich human-computer interaction, but also a reusable, portable system. This system could greatly improve the coverage of the forecast information, use a common browser to release geographic information, greatly reducing the costs of training and technical burden of end customers, making not only professionals, but ordinary users can easily obtain the required information. WebGIS is easy to integrate seamlessly with other information services in the Web, establish multi-functional and specific GIS applications, realize the goal "once compiled, run anywhere", and really take the advantage of cross-platform. In the future, we will do some follow-up study to achieve to load the deployment of local vector map. According to the XML feeds provided by authoritative department, complete the application which meets users' actual needs.

\section{Acknowledgements}

This work is partially supported by the Jiangsu Provincial Industry-Tech Program (BE2011195). 


\section{References}

[1] Greneker, E.F.; Geisheimer, J.L. Mapping Lightning Channels in a Thunderstorm by Radar[M]. Aerospace and Electronic Systems Magazine, 2003 , Page(s): 4 - 7

[2] Huijun Li. Silverlight Perfect Journey[M]. Beijing: Publishing House of Electronics Industry,2009

[3] Hailong Yu. Study on WebService-based Application Model Integration[J]. Surveying and Mapping,2006,35(2):153-159

[4] Ye Jiadong, FanPeifeng. Mathematical Methods of Artificial Weather Statistics[M]. Beijing,Science Press,1982:197-245

[5] Liang Guo, Jianhua Gong, Jun Sun,etc. Study on GIS architecture based on SOA and RIA. Information Sciences and Interaction Sciences (ICIS) Conference, 2010 , Page(s): 620 - 625

[6] Xin Qu, Min Sun,Chen Xu,etc. A Spatial Web Service Client Based on Microsoft Bing Maps Geoinformatics 2011 19th International Conference, 2011, Page(s): 1 - 5

[7] Yu Zhang. [Silverlight] Bing Maps Articles[EB/OL]. http://blog.csdn.net/beniao277/archive/2010/01/3I/5273I09.aspx,2010-01-30 